Biology | Sung-Hou Kim

\title{
Rethinking the Tree of Life with new tools
}

Professor Sung-Hou Kim and his colleagues from the University of California, Berkeley, have applied a new way of thinking the tree of Life, a concept that has been around since Darwin's time. Drawing from applied an Information Theorybased non-alignment method to compare whole-proteome sequences, the protein sequences coded by all genes of each organism. Their research group has studied over 4,000 organisms and their results show vidence of a 'deep burst' of the founders of all six major groups the Tree of Life (Figure 1).

o understand and help us to
visualise the connectedness of
life on Earth, scientists construct
phylogenetic trees. A phylogenetic tree
of all organism groups, or 'Tree of Life',
is a branching diagram that shows the
relationship between organisms based
on certain characteristics (Pace, 2009).
The great naturalist, Charles Darwin, first
sketched his ideas about how species
might evolve through time (Darwin, 1859 .
His ideas quickly came to symbolise the
theory of evolution by natural selection
(Figure 2). Organisms that are more
closely related are found closer together
on the tree. For example, a human and
a chimpanzee would be situated close
together; a wolf and a shark would be far
apart. The Tree of Life is still used today,
as a model and research tool, to explore
evolutionary relationships and to provide
a simplified narrative of evolutionary
history, which itself continues to evolve.
Understanding the connectedness
between species helps us to answer
important broad scientific questions
such as 'How and when did all $\begin{aligned} & \text { Last branches in rey } \\ & \text { Leaf nodes around EPS=100 Bacteria }\end{aligned}$
$\begin{gathered}\text { such as 'How and when did all } \\ \text { diversity of Life emerge? } \\ \text { or more specific }\end{gathered}$
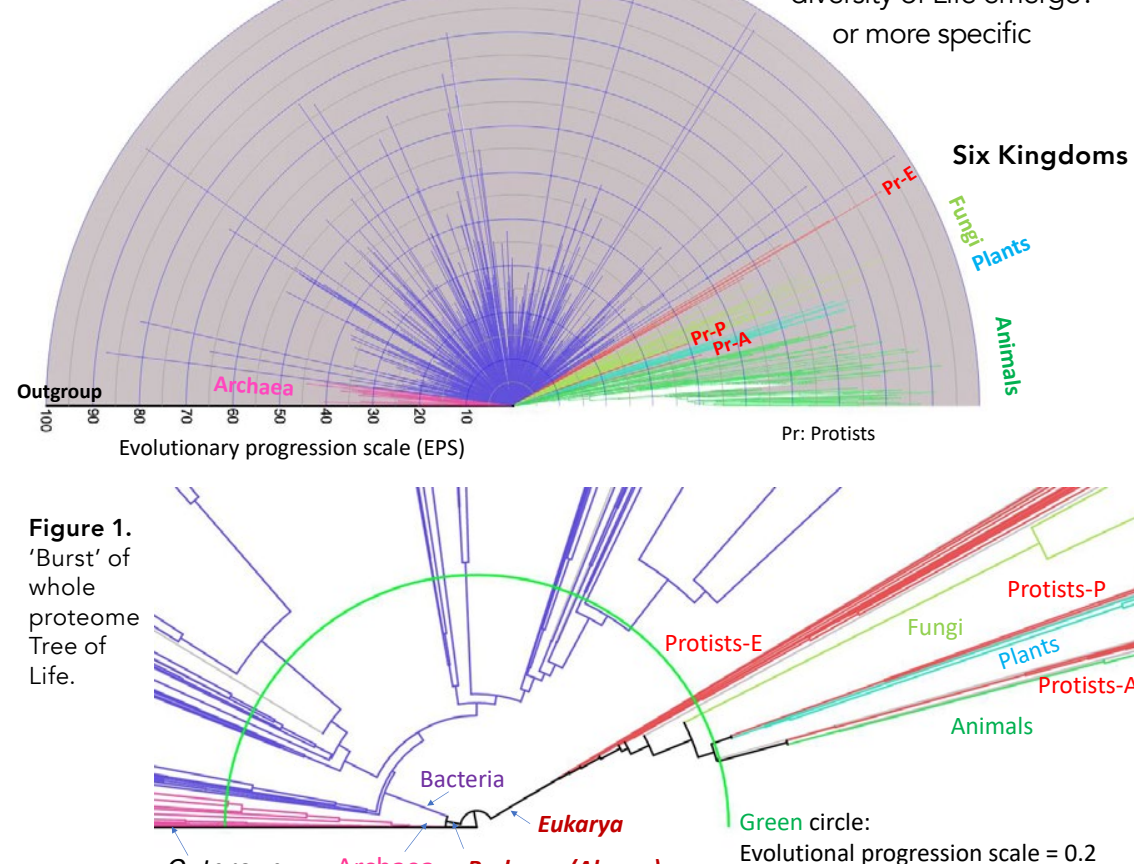

questions such as 'How did HIV evolve? or the currently urgent question 'How did 19 virus emerge and how are its variants evolving?

\section{REVISITING THE TREE OF LIFE} INTHE GENOME ERA As a concept, the Tree of Life gives complex picture that is vilution important to remember that a Tree of Lfe is not an actual record but a model an approximation of life's history. Damin himself wrote about evolution and ecosystems as a 'tangled bank'. As new data become more comprehensive, and our tools and methods to compare organisms become more sophisticated, however, so do our approximations.

In Darwin's time, a Tree of Life was constructed by comparing the external morphology of organisms. Later, interna skeletal morphology was introduced, yielding richer insights into organisms degrees of affinity. These methods ccintists to mart to orogy helped scientists to start to organise the 'big of all organisms.

Following advancements in molecular biology, evolutionary biologists applied analysis of selected genes to describe the are made up of DNA (deoxyribonucleic acid) and are the main unit of heredity. Variations in gene sequences offers another way to shine a light on

evolutionary variation. Most genes act as codes to make proteins, the workhorses of all cells; for example melanin, which influences eye or skin colour in humans and is passed down through many generation leading to differences mutatween organisms. Woese and Fox (1977) turned the Tre Wose and Fox (1977) tumed he Tree sequences of parts of ribosomes (the cellular machinery hat make proteins in 'trunk' to the Tre of lif added a third discoven of the third domain Arche (distinct from bacteria and eukaryotes). Importantly this discovery highlighted the fantastic diversity of microbial organisms. We now appreciate that these single-celled organisms represent the vast majority of the Earth's genetic, metabolic and ecological niche diversity including hot springs (Figure 3).

\section{GENE OR PROTEIN}

\section{ALIGNMENT METHODS}

Constructing an organism's Tree of Life using only gene sequences has its ow limitations. It gives just a glimpse of evolution based on that particular gene for only very smalt frat, which account for only a very small fraction of all genes apply in using only a set of select protein sequences to construct a Tree of Life. Until now, the most common methods of constructing Trees of Life using a set of select genes or proteins from the wholeenome sequence require sequences to be lined up. For this reason, they are called alignment-

A phylo

A phylogenetic tree, or a Tree sequences of genes of A phecicic topics to as a branching diagram that shows the the reader and similar alludy organisms are is a branching diagram that shows the books for example all compared after they relationship between organisms based books written by the aligned. All alignment- on certain characteristics. based methods look for similarities between the sequences within only the aligned regions and use these to calculate relatedness.

More recent advances in phylogenetic analysis (the study of evolutionary (ationship of organisms) have used a set genome sequencing, where the alignable genes account for a small fraction of the whole genome. A genome is the entire genetic material of an organism, including all genes coding for proteins and RNAs as well as non-coding regions. As much as $99 \%$ of the human genome made up of non-coding portions of DNA Nenter et al, 2001).

Although the alignment-based methods have been the most common ways several decades, however, ha
California, Berkeley applied a novel aproach to sequence comparisons for without alignment. The researchers took inspiration from computer algorithms used in Information Theory to compare books without alignment of words. frequency profile', which is a collection and their frequency in each book, to estimate the degree of 'divergence' between two books without aligning sentences or paragraphs. Professor Kim team adapted this approach to compare whole-genome or whole-proteome sequences without the need for selecting them for alignment at any stage in analysis. A major benefit of alignmentfree me A major be hathey can be used to genomes or proteomes.

\section{THE TREE OF 'BOOKS'}

Tree of developmental step taken by Kim and his colleagues involved English literature. The words of a book can be considered genome sequence of

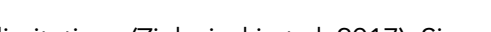
only a small fraction of wholeg nines only a salined this tells us about the .

\section{A NEW VIEW:}

ALIGNMENT-FREE METHODS and his colleagues at the University of of all unique words (letters in context)

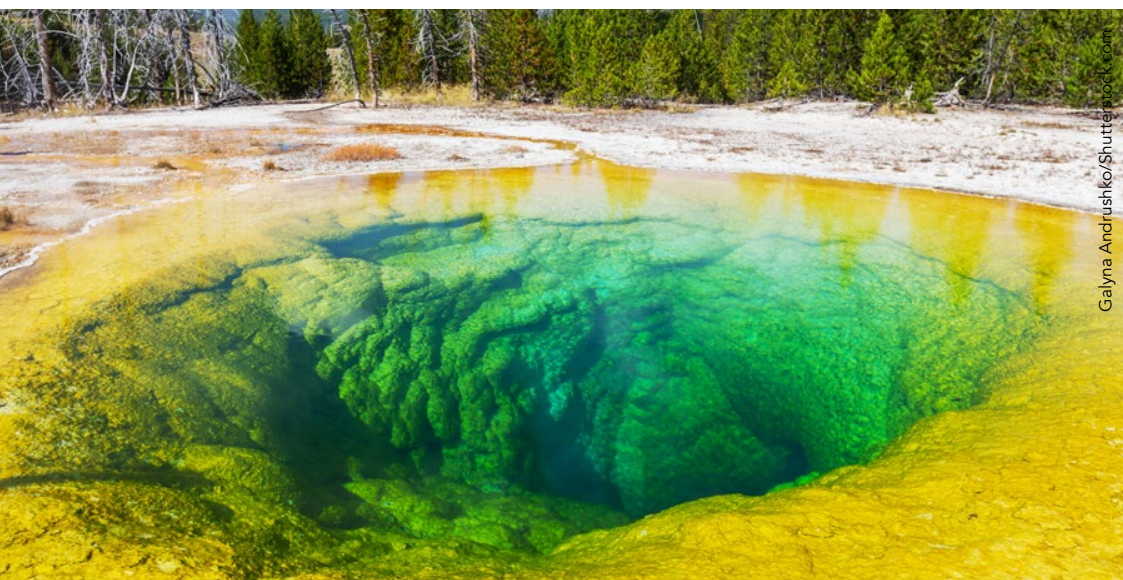




\begin{tabular}{|c|c|c|c|c|c|}
\multicolumn{2}{c|}{ A B B A B A } & \multicolumn{2}{c|}{ B A B A B A } & \multicolumn{2}{c|}{ D E G F E E } \\
\hline ABB & 1 & & & DEG & 1 \\
\hline BBA & 1 & & & EGF & 1 \\
\hline BAB & 1 & BAB & 2 & GFE & 1 \\
\hline ABA & 1 & ABA & 2 & FEE & 1 \\
\hline
\end{tabular}

Figure 4. With a feature frequency profile (FFP) using a feature length of three, we see that two of a content of the alphabets and their contexts. The frequency of each unique feature in the 'book' was then counted. Thus, the collection of all unique features and their frequencies, called 'Feature Frequency Profile' (FFP), yields all the information needed to reconstruct the original 'genome book'. Using various mathematical operations each 'book' as an FFP vector can easily be compared to different 'books' and

a tree of 'books' can

case; if we build an FFP using a feature length of three, we can see that two of the strings share features, but with different frequencies (Figure 4).

Thus, the feature length plays an important role in telling similar character strings apart. The ideal feature length has the highest number of different features that can distinguish each string.

Archaea, Bacteria, Protists, Fungi, Plants, and Animals), or 35+ 'minor major group, the Protists (single-cell Eukaryotes), subdivides into three types: the first emerging at the basa

position to all multi-cellular eukaryotes; the second and the third at the basal positions to all plant and all animal major groups, respectively Figure 1 ).

On the order and the progression stage of emergence of the various groups, the differences are much more pronounced and unexpected: 1) life started by the

This method can shed light on a founders of two domain previously understudied evolutionary $\begin{aligned} & \text { Akarya (Prokarya) and } \\ & \text { Eukarya simultaneously, }\end{aligned}$ Once all the trees were phenomenon: the "deep burst" of life. Prot sequentially from
constructed for a wide constructed for a wide ange of the lengh of (n) topology by identifjing two adjoent trees (with the feature-length difference of one) that have minimal topological difference. The optimal tree of the 'books' revealed that similar books (author/ topic/genre) were grouped together (Sims 2009a). Encouraged by the result, they tested the applicability of their FFP method on living organisms: Kim and colleagues repeated the process using whole genome sequences of some of the best characterised mammals (Sims 2009b) with good agreement

A note about the properties of 'features': Let us look at three different character trings contaning two difterent strings possible feature length is one and the longest possible feature is six If the feature length were one, the FFPs of the strings containing As and Bs would be identical because they each contain the of each letter. However, this is not the

SHAPING THE WHOLEPROTEOME TREE OF LIFE the BFP method one step further by considering whole proteomes (the complete set of proteins encoded by all genes). They have now completed analysis of the proteomes derived from whole-genome sequences of over 4,000 organisms available in the public genome database (The National Center for Biotechnology Information. National Institutes of Health USA). Their FFP tree shows many similarities to the historic morphological and convention alignment-based gene Trees of Life in grouping pattem, but some fascinating and somethes radical differences in the groups (Choi, Kim, 2020).

On grouping, the whole-proteome FFP tree of life suggests: 1) All organisms studied assort into two 'trunks' (domains of 'Akarya' (Prokarya) and Eukarya) six 'major branches' (kingdoms of

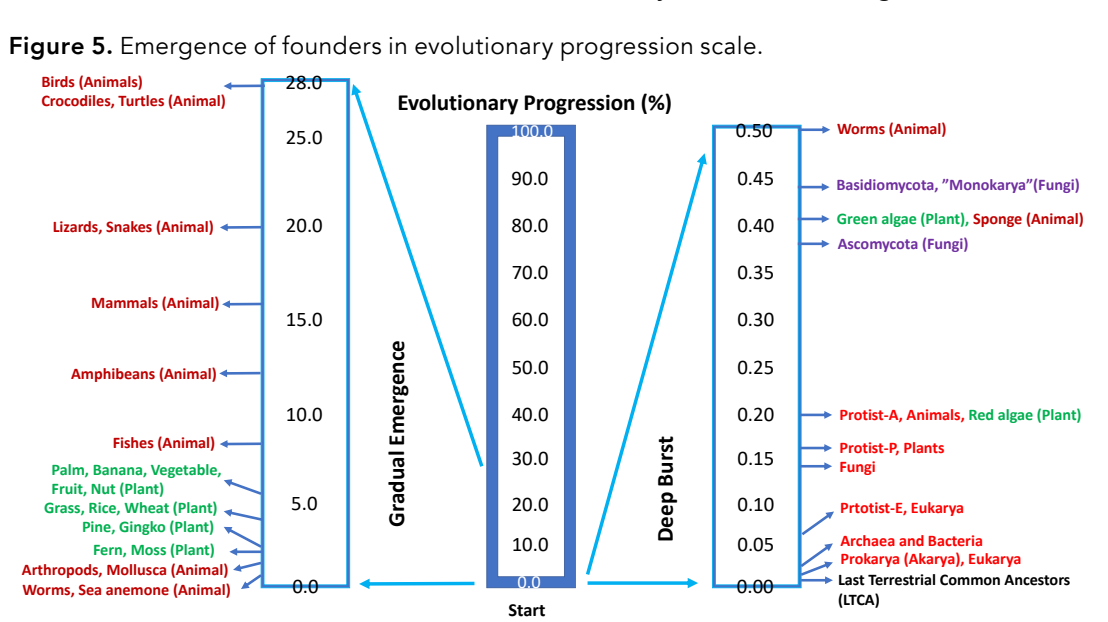

alignment-based trees: 2) there was

a staged 'deep burst' of organism

diversity, where the founders of all six

major branches of all living organisms

emerged within the first $0.20 \%$ of the

entire progression of the evolution of life

(Figure 5). Following this, a more gradual

and step-wise evolution seems to have taken place for the remaining $99.80 \%$ of the evolutionary progression scale, simila to the evolution by more gradual natura selection that was described by Darwin. Kim's group found in their finding of the staged 'deep burst' of life's diversity, some similarity to the 'Big Bang' theory of the origin of the universe as suggested differences. Kim and with some key therences. Kin and his colleagues of all major groups of insects near the roo of the insect tree (Choi, Kim, Kim, 2020). The team is now focusing its research efforts into the detailed features of the whole proteome trees of other major groups and minor groups.

Our image of the Tree of Life has been evolving with the explosion of new whole genome sequences and the evolution of the scientific tools and methods used to study it. With their alignment-free methods, Kim and his team at Berkeley are challenging the way we construct our evolutionary history once again. Their use of massive whole-genome information and an ability to think outside the box is challenging the status quo and diving our exciting new places.

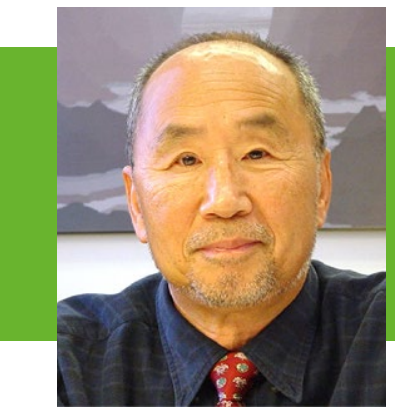

\section{Behind the Research}

\section{Professor Sung-Hou Kim}

E: sunghou@berkeley.edu T: +1 510-08 2564 W: chemistry.berkeley.edu/faculty/chem/kim

Research Objectives

Professor Kim's group applies computational genomics to glean a wide-angle view of all or specific groups of living organisms.

\section{Detail}

\section{Address}

Department of Chemistry Berkeley, CA, 94720, USA

Bio

Kim is a faculty member at the Department of Chemistry and Centre

\section{References}

Choi, J, Kim, SH, (2020) Whole-proteome tree of life suggests a deep burst of organism diversity.
doi.org/10.1073/pnas.1915766117

Choi, J, Kim, BJ, Kim, SH, (2020) Whole proteome Tree alignment. bioRxiv, doi.org/10.1101/2020.07.11.198689 Darwin, CR, (1859) On the origin of species by means of natural selection, or the preservation of favoured races in the struggle for life. London: John Murray. [1st Edition] Koonin, EV, (2007) The biological Big Bang model for the major transitions in evolution. Biol Direct, 2, 21. doi.10.1186/1745-6150-2-21

Pace, NR, (2009) Mapping the Tree of Life: Progress and Prospects. Microbiology and Molecular Biology Reviews, 565-576. doi.org/10.1128/MMBR.00033-09 Sims, GE, Jun, SR, Wu, GA, Kim, SH, (2009a) Alignment-free genome comparison with feature frequency profiles (FFP) and pnas. 0813249106

Sims, GE, Jun, SR, Wu, GA, Kim, SH, (2009b) Whole-genome phylogeny of mammals: Evolutionary information in genic
and nongenic regions. PNAS, 106 (40), 17077-17082. doi. org/10.1073/pnas.0909377106

Venter, JC, et al, (2001) The sequence of the human genom
Science, 291, 1305-1350. doi.10.1126/science.1058040 Woese, CR, Fox, GE, (1977) Phylogenetic structure of the prokaryotic domain: the primary kingdoms. PNAS, 74 (11): 5088-5090. doi.10.1073/pnas.74.11.5088 Zielezinski, A, Vinga, S, Almeida, J, Karlowski, WM, (2017) Alignment-free sequence comparison: benefits, application, nd tools. Genome Biology, 18:1-17 for Computational Biology, University of Funding $\begin{array}{ll} & \text { Gitt fund } \\ \text { in structural biology and computational } & \text { Berkeley }\end{array}$ Acadics. Menber of the US National ( (- Bellin Choi - JaeJin Choi

ponse

\section{Personal Response}

\section{What sparked your interest in phylogeny?}

II My research interest started with my desire to get detailed views of the 3D structures of individual protein
and nucleic acids to find their architectural motifs and their relationship to their respective functions. After some successes, my interest expanded to get a view 'universe'. Finally ly recognising throtein structural $3 \mathrm{D}$ structures of proteins are encoded in their amino acid sequences, my interest expanded further to find ways to get a 'wide-angle view' of all organisms, each represented by their proteome or genome, and to find
possible evolutionary relationships among them.

\section{Do you think your background in crystallography gave} you a different perspective?

II In crystallography, the more comprehensive (high view of the whole molecule one gets. Thus, to construct a new organism Tree of Life, I wanted to start with the most comprehensive data available for each organism proteome sequences. 